\title{
A CONCEPTUAL FRAMEWORK FOR THE SCAFFOLDED MIND AND ITS INFLUENCE ON ENHANCING MULTISENSORYEXPERIENCE IN CORPORATE IDENTITY
}

\author{
Mirette Gamal Kamil MOAWAD, Nagwa Yahya EL-ADAWY, Dina ABBOUD *
}

Department of Advertising, Faculty of Applied Arts, Helwan University, Egypt

\begin{abstract}
The various cognitive processes are rooted through the interaction of the individual towards the information coming to him from the surrounding environment with the cognitive balance and experiences that result in the actual experiential perception of his previous scaffolding processes. This stimulates mental support processes by creating implicit links between concepts and symbols and extrapolating from the meaning of what they narrated. This contributes to enhancing embodied cognition in the design of the corporate identity, and drives the impulses to mental persuasion, and then Multi-sensory experience, stimulating the unconscious response of the recipient. Hence, the research problem is summarized in how to employ mental support to discover the relationship between metaphorical concepts and the meaning to be communicated to enrich design. This study aims to revive the cultural and artistic heritage through conceptual knowledge, and to support it by developing, modifying and consciously borrowing from different cultures with a new perspective. This achieves knowledge exchange, and reflects positively on the upgrading of the Glocal market value of the corporate identity in the global local markets. The research follows the descriptive analytical approach by presenting some modern graphic techniques based on mental support to enhance the sensory experience in design. One of the most important results is to shed light on the effectiveness of the supportive mind in enriching meanings and values and upgrading them to inspire new concepts, and this study concludes the importance of setting standards on scientific grounds by employing sensory features in design elements such as symbols, decorative patterns, textures and others in a tight artistic consistency to benefit from the influence of the mind Supporter in discovering the content of the advertising idea and deepening its mental image in the memory.

Keywords

Multi-Sensory Experience, Embodied Cognition, The Scaffold Mind, Exaptation, Conceptual Knowledge, Multi-Sensory Experience, Global Markets
\end{abstract}

\section{Introduction}

Mental processes are also physiological because they deal with the surrounding environment, as no cognitive or movement activity can be carried out except through them after classifying them to find out the differences between each of them and the extent of the individual's response and perception of visual or tactile stimuli and others after processing and storing them in memory to be called upon when needed. From here it is possible to shed light on what the supportive mind is and its effect on experiential knowledge in order to consolidate concepts and meanings while creating implicit links between them, and the importance of activating mental support with sensory stimuli and intellectual drives is due to their role in stimulating the unconscious response of the recipient through the processes of adjustment and the permanent development of aesthetic and functional features in the design; To give it a distinct character. According to quality standards. The use of symbols, signs, and

* Corresponding author: appliedarts@a-arts.helwan.edu.eg 
visual or verbal metaphors is one of the important means of communication in the modern era, with which many civilizations and cultures correspond to it, and therefore it is a bridge between them. They can also be used in graphic designs and decorative patterns because they contain the meanings that support the cultural heritage and revive it in a modern way appropriate for the era. Mechanisms for transferring conceptual knowledge were presented as a basis for mental support and enhancing its impact in order to present a message that has a meaningful and integrated context that achieves its goal at the individual and collective level. With the help of experts specializing in knowledge management that is based on achieving adaptation and standardization with scientific and technical consensus to make conscious use of civilizations and human heritage and promote it to reach a competitive position in all technical fields in general and enhance the value of the corporate identity in the local and global markets in particular.

\section{Statement of the Problem}

We still need more effort in determining how to benefit from the influence of the supportive mind in creating links between the elements of corporate identity design and the values and symbols it contains with implicit meanings. Which contributes to enriching conceptual knowledge and enhancing the sensory experience of the recipient, and from here the research problem is summarized in an attempt to answer the following questions:

How can mental support methods be employed to enrich knowledge and revive traditions and cultural symbols in design?

To what extent does the supportive mind contribute to enhancing the multiple sensory experience in designing the corporate identity?

\section{Research Aims}

The research aims to:

- Shedding light on the influence of the supportive mind in linking values and symbols with the connotations and meanings implicit in designing the corporate identity; And then enhancing the sensory experiences and consolidating the mental image of the recipient.

Employing the enhancement of the physical properties in the design or reformulating them to add new plastic dimensions; Which reflects positively on creating different creative ideas with both aesthetic and functional values.

\section{Significance:}

The importance of research is due to:

Focusing on how to activate mental support processes to stimulate the sensory response of the recipient, by developing the design by introducing new additions or reinforcing it with 
symbols to revive the traditions and cultural heritage; Thus achieving the competitive advantage of the corporate identity and upgrading it in the local and global markets.

Hypothesis: Research Hypotheses

The research assumes that:

Activating the support processes contributes to stimulating the subconscious mind to derive metaphorical concepts and decipher them in order to reach the intended meaning in the advertising message. And then triggering the impulses by the sensory response of the recipient.

The transfer of brands from the local markets to the global markets by employing the symbol as a tool for cognitive support that contributes to achieving cultural exchange and enhancing its competitive advantage by becoming a bridge linking them.

\section{Hypothesis: Research Hypotheses}

The research assumes that:

Activating the support processes contributes to stimulating the subconscious mind to derive metaphorical concepts and decipher them in order to reach the intended meaning in the advertising message. And then triggering the impulses by the sensory response of the recipient.

The transfer of brands from the local markets to the global markets by employing the symbol as a tool for cognitive support that contributes to achieving cultural exchange and enhancing its competitive advantage by becoming a bridge linking them.

\section{Methodology:}

The research follows the descriptive and analytical approach by presenting some modern designs in the corporate identity that depend on employing mental support methods.

This is to know its effect in enhancing the sensory experience of the recipient.

\section{Research limits:}

The research is determined by focusing on the application of mental support methods in designing packaging with multiple sensory features as one of the important elements of corporate identity.

\section{Terminology: search terms}

- Scaffolded mind: It is the presentation of a specialized "niche construction model" from cognitive sources supported by societal experiences while activating it creatively through the use of verbal, visual and formal metaphors. In order to consolidate concepts and enhance capabilities in the fields of arts, architecture and others. 
- Multi-sensory experience: It refers to perception through the physical senses through effective sensory stimuli that have the ability to attract attention, create the impression and evoke a distinct mental image in the individual and build and strengthen the connection between him and the exhibit.

- Conceptual metaphor: It is a distinct cognitive mechanism in which mental maps of the connected connections between elements of non-similar concepts are drawn by employing the imagination factor to understand its significance.

Exaptation: it is a term referring to a new pattern in the use of knowledge in order to develop the initial features of the product to give it functional characteristics related to arts, culture and others. Which enhances its capacity and diversity of uses.

Glocalization: It is a term referring to the conscious convergence between the local and the global as a result of the processes of knowledge exchange and the investment of creative energies while making use of modern technology in creating bridges between the local and the global, provided that the national identity is preserved.

\section{First - Levels of mental cognitive processes:}

Perception and behavior are based on a basic base, which is knowledge that provides the mind with a wide range of experiences. Perception: Actual perceptions are a result of friction and interaction with things physically and morally, and this leads us to classify knowledge from a modern perspective according to scientific studies specialized in developmental psychology and nerves, which are as follows:

1. Basic knowledge: "Grounded cognition" and it is the most widely circulated because it refers to the constants in the material and objective world around us, and it is considered one of the most stable types of knowledge.

2. Embodied cognition: It is based on the genetic development of the individual and the degree of his response to the objective physical characteristics in addition to the "ontogenetic development" which comes as a response to a sensory stimulus followed by an appropriate motor reaction to it; "Sensorimotor" sensory-kinesthetic experiences are thus considered more Species change, evolve and grow.

3. Present / real knowledge "Situated cognition": It refers to the method of processing information and concepts, storing them in memory and recalling them if needed. It depends on the chronological and social context contained in it and is therefore called "contextual information."

This knowledge is characterized by dynamism and flexibility towards any developments, whether personal, environmental or otherwise. 
As it deals with it according to mental functions such as causal, intentional, or analytical reasoning. Therefore, experiential knowledge is considered the link between the objectivity of "basic perception" and the dynamic change of "present knowledge". It should be noted that the previous classification is theoretical for the study only.

Because in practice these three concepts of knowledge overlap in perception, behavior and experiences according to circumstances, attitudes and the degree of cognitive construction of the individual.

\section{Second: What is the supporting mind and its effect on (actual) experiential knowledge:}

1- The concept of a supportive mind: "Scaffolded mind"

The term "consolidation" is used to refer in some of its aspects to the consolidation of concepts and knowledge through various sources (historical, cultural, artistic, heritage ...) in addition to the recognized symbols, and it has a vital role in the cognitive development of the development of an individual's thinking; the wider the circle Available knowledge from the outside world has deepened forms of mental support as the scientist sees a mindset with an ability through which the information received by him is combined with the basic cognitive structure accumulated in him since early in his life with perceptual and kinetic systems in which the subconscious mind stimulates the unconscious response supported by the mind and results in perception.

Experimental by adding previous experiences to the modern in order to obtain a better and more comprehensive understanding of the actual "embodied cognition" of the concept of consolidation.

"Heuristic value" for information and knowledge, which is called the inferential value and activates the supportive mind with multiple sensory stimuli and the intellectual and life drives that stimulate it to play its active role in linking Al-Dhamani values, concepts and symbols.

2- Between the scaffolded mind and the extended mind

If the supportive mind refers to the distinctive cognitive construction process by gathering information resources and activating them in an innovative way through the use of metaphors, formal symbols, and interesting coding in written phrases through the transfer of ecological experience and technologies, then the extended mind is part of intergenerational social learning, which is called learning Intergenerational social is limited by the supportive mind that picks up signals and notes quickly and with a wide range; But without analysis, interpretation and deduction, as happens with the supportive mind, which is more comprehensive and deeper because it provides the conceptual tools to complete the "transformative processes" by creating connections and applications between the visible and 
its meaning, which deepens the cognitive competence and enriches the mental and psychological capabilities to reach To experiential awareness by stimulating the subconscious response to emotional stimuli with social, cultural, and sensory standards that individuals interact with in order to reach the goal of changing attitudes for the better and upgrading individual and social behavior.

\section{Third: Forms of scaffolding}

\section{1- Genetic Support: "Ontogenetic Scaffolding"}

It is the first form of consolidation that arises very early and spontaneously from childhood through the conceptual development acquired throughout life.

This is a natural result of continuous interaction with the family and the surrounding environment.

\section{2- Support by development: "Phylogenetic Scaffolding"}

It is called "Evolutionary scaffolding" and the scientist "Williams" considered it essential in the advanced sciences of human knowledge by selecting the best through good treatments "Good tricks" in order to support many abstract concepts and meanings with alternatives to recognized symbols. For example, the sign of peace with an olive branch, the hand as a symbol of work, and the shape of the heart to indicate love, intimacy, and so on.

\section{2-Reinforcement by contour: "Exaptation"}

It is an application of this type of support by development, and this term refers to a process of selection of some characteristics that are reformulated with new additions that enhance their capacity and efficiency in design that combines the previous features with their modernization, which qualifies them for another work. Thus, this product achieves a multiplicity of uses with the help of environmental and technical factors, and this is reflected in its functional diversity. "Contour" is a process in which changeable features are redistributed with the aim of bringing about a qualitative or functional shift for a goal different from its original function. It helps to create creative atmospheres with multiple dynamic levels of innovations that lead to many exciting discoveries.

Both worlds "Gould and Vrba" consider that the aesthetic and functional characteristics that are formed through selection or adaptation processes from original use are called "effect" by introducing other characteristics that develop from the applications of the idea or product in different areas of life; As happens in architecture using what is known as spandrels, which is a term that means the process of altering some shapes, and spaces to design innovative decorative works borrowed from them; Such as the intersecting arches in the corners, taken from the shape of triangles, which are fixed in the corners of the ceilings as decoration; Thus, 
through the development support, it was able to create other functional features, and to use them in combination for a new use, which is called "Co-opted for a new use".

A narrow exaptation, which is when the development is in the same field or sector and the other type, is extensive exaptation, and is used in divergent fields and sectors different from its original scope.

\section{3- Cultural scaffolding.}

Both Salomon and Perkins defined cultural consolidation as involving the control of cultural tools and the resulting cultural and artistic works that include many sources of information, values and concepts. Therefore, design elements related to historical and heritage aspects Religious and other support individuals intellectually, emotionally and socially by circulating and transmitting that knowledge of its cultural characteristics among individuals; Which establishes its pillars intellectually in society and thus is considered another source

) To develop cognitive processes and moral values by strengthening effective community participation.

\section{3- Symbols as scaffolding:}

Many aspects of human and cultural nature depend on symbols in the fields of language, science, the arts and many other activities, and they are intrinsically linked to the cognitive development of the features of the things they symbolize.

The metaphorical consolidation refers to how the symbols are used in building other meanings that are more established and permanent, and thus are considered part of the permanent cognitive development of the fields, relying on the generality and inclusion of the semantics of some symbols as an agreed language across different cultures, a feature that is specific to them.

Which makes it suitable to be a common basis to build upon, and it includes signs, signs, and letters that humans use as tools of communication and interaction and passed on to generations as a human heritage of peoples. Symbols are elements in the language of ideas known as the "standard view". Its consequences have a specific interpretation in the thought of the sender and the recipient and the consequent learning and cognitive support "cognitive scaffolding" by stimulating ideas and imagining implicit meanings behind them, which contributes to Creating new values.

The trademark "VIA RAIZ" is a company that specializes in designing modern products for some professional trades such as textiles, ceramics, and home accessories in Mexico; The design idea is based on the concept of "Cultural Origin", with the intention of reviving its civilizational roots. Symbols that have special connotations in cultural heritage, popular art 
and some myths associated with meanings and values such as eternity, optimism and others were used in this, as they refer to the most prominent tourist attractions in the country. It has expressed signs indicating some craftsmanship on its products to confirm the extent of pride in its heritage that distinguishes it so that it will be perpetuated for successive generations and transmitted by other cultures across continents. These icons are depicted as a mosaic in a wonderful artistic way that combines various activities and artistic skills.

\section{Fourth: Dimensions of scaffolding.}

Of the most important basic dimensions of mental support are as follows:

\section{Reliability: Trust}

The recipient's confidence in the sources from which information and data are drawn from the design and advertising elements is an important pillar in creating his credibility, especially if there are many and varied methods to serve a specific goal, which leads to a sense of transparency and familiarity towards it.

Research studies have confirmed that reliability is directly proportional to The degree of response of the recipient whenever more influential resources are presented from an emotional and human aspect to stimulate his senses by responding and interacting positively with it.

Which reflects on his psychological state and mood with joy and enjoyment while renewing his activity and vitality.

And this comes by investing all the available resources from the beauty of nature, music, arts, metaphorical meanings, and abstract values such as love, goodness, giving and others.

It is worth noting that the emotional response of the individual is achieved most in the social atmosphere, such as celebrating events and interacting with others through mechanisms called "Bottom-up", which is an approach to emotional control that has Emotional control that follows the theory of cognitive behavior and depends on the organization of influences and their immediate treatment at the level of the unconscious first, then Then it is perceived and verified mentally with awareness and attention; This is in contrast to the "top-down" approach, which is perceived and controlled by acceptance or rejection before being affected by it.

The importance of knowing this theory for scientific benefit is due to the fact that the affective influence strongly supports the vital interaction of individuals with what is presented to them by the unconscious response, which then takes root in the form of a subjective sensory experience. 
Philosophy of America, which specializes in the production of cosmetics, skin care creams and perfumes from natural sources, is inspired by the selection of the name of its products, a brief philosophy in expressive words that summarizes the effect of its exhibits honestly reflects the confidence of its customers from their previous experiences, for example but not limited to choosing metaphors with meaning such as " Time in a bottle "; In other words, we will save you time with this product, and "Renewed hope in a jar with the intention of renewing hope for a fresh skin against the signs of time," and also "Full of promise," meaning that this product is full of good promises, and so on to encourage its customers to acquire these products due to their reliability and safety of their components.

2- Personalization and Reinforcement: "Individualization and Entrenchment"

This dimension is related to the former, as trust is the basis of personal interaction with sources that have credibility with him, and the emotional influence creates ties at the individual level, which then extends to others around him through the exchange of previous experiences that aim at continuous development to reach the best application by quoting and adding to be achieved. Technical and technical support for the exhibitor.

This dimension depends on distinction, modernity, and the dynamism of the information or the excitement, all within a specific time frame, and it is also of a high degree of impartiality and excitement at the same time until settle into the consciousness of the recipient; To strengthen the sense of it due to its enjoyment of what is called "performative entrenchment" by providing a product that has unique features and characteristics that affect the human being and meet his needs in this field.

The trademark "Give-A-Care": is about a company in Canada that produces medicinal preparations for women with breast cancer.

The idea of designing the identity was based on taking into account their health condition while providing psychological support with support and spreading the spirit of hope in strong and influential terms that address their actual needs to pass this critical stage safely.

And safely; And that in terms on the packaging of their products that have a distinct personal character that confirms the extent of attention to the smallest details of their lives in order to alleviate their suffering, for example presenting sweets equivalent to the taste of bitterness during and after the use of chemotherapy with an advertising phrase that supports their determination saying:

"When life gives you lemons and those lemons taste like chemo you need candies"

With the presentation of a bag that gathers everything they need, coupled with positive expressions of encouragement, they are in the focus of attention and thinking. 


\section{Fifth: Metaphor as a source to support conceptual knowledge in design:}

"The metaphor as a source for scaffolding the conceptual knowledge in design"

1- Conceptual metaphor

Myths and verbal metaphors are fertile material in creating emotional feelings and linking written and verbal cultures through the combination of truth and fiction with Jaraphic treatments in designing the corporate identity that makes it go beyond being a mere sociocognitive mechanism to an advertising message with meanings and contents that serve the idea and purpose of the design, In this, they take actual "embodied modes" in the form of symbols, logos, and figurative patterns with agreed connotations.

The word "Metaphor" is due to the Greek origin "Meta", meaning behind, and the word "Pherein" means briefly.

Metaphors are a way of seeing something as if it were something else and then finding a bridge between two different fields by employing the accepted context with artistic manipulation in order to build a meaningful sensory experience that touches the conscience.

Therefore, metaphorical concepts are not just a hollow linguistic embellishment in the advertising phrase of design, but they allow the imagination to pick up signs and discover the link between shapes and symbols with abstract meanings and values.

Which strengthens the mental image of the recipient. It should be noted that metaphors of various kinds, from verbal, visual, kinesthetic and others, can be circulated through the use of images, colors, shapes, textures and other design elements that stimulate the multiple sensory experience.

\section{The role of metaphors in shaping corporate identity:}

Metaphors can be used in the elements of corporate identity as a means of communicating a huge amount of encrypted information with its vague meanings in a way that entrenches some concepts in the advertising message.

Whereas, the experimental metaphor in the elements of identity is considered a window between different cultures by stimulating rapid interaction with them through logical thinking in understanding their connotations and the shared values they indicate; This is reflected in the creative energies of the designer in creating innovations that enhance the sensory experience by using symbols, graphic patterns and others.

The expressive power of metaphors lies in the presentation of innumerable ideas and meanings as it is a fertile field in igniting the imagination and addressing the subconscious first because of its "compact", targeted and attractive information in the advertising message that keeps pace with the age of speed. It also contributes to the cognitive convergence 
"Conceptual glue" through Creating emotional ties with the corporate identity by using a team specialized in the field of marketing and advertising based on this mediator in the design elements in order to achieve long-term results that achieve the competitive advantage of the corporate identity and give it an added value that goes beyond space and time.

\section{Using visual metaphor as a method of reinforcement:}

The visual stimuli such as the shapes, decorative patterns and colors used in the advertisement are a metaphor for a specific message that the designer aims to send to the recipient to achieve the effective effect of the advertisement.

As visual thinking is more modular and less stereotyped than verbal thinking. The fertile imagination plays an effective role in modifying, analyzing, organizing, and reformulating visual images into new creative forms that constitute a visual sensory experience for the recipient.

\section{Metaphor enrichment of social knowledge:}

We consider in this research study the close relationship between the tactile sensations recorded in the conscious mind, and between abstract ideas and values, and specific personal attitudes, so we describe these with those spontaneously and with those familiar in social life. For example, we find that the tactile characteristics of things such as warmth, hardness, softness, etc.

We call it verbs to reinforce their meanings, so we say: "He faces a hard decision," or "His feelings have cooled." And so on, such as Deep meaning or Shallow thinking, all of which are descriptions of sensory patterns.

"Different but has a linguistic connotation that stimulates the subconscious emotional experience; This confirms that the metaphorical concepts are strongly reflected in the language in which the title of a book, a piece of music, or an advertising message, and so on, is formulated.

\section{Results:}

1- Activating methods of mental support in design that contribute to refining sensory experiences and promoting the cognitive growth of the recipient.

2- The allegorical reinforcement is highest when there is an unusual verbal conflict. Which stimulates the subconscious mind to deduce the hidden meanings to be conveyed in the advertising message.

3- Supporting development in design or modification of some sensory features opens up broad horizons for fertile imagination and innovation, and reflects positively on the creation of many creative ideas with aesthetic and functional values. 
4- The importance of employing symbols and the common values and concepts they bear, which contribute to the revival of traditions and cultural heritage while strengthening effective community participation.

5- Achieving the reliability factor in the design in order to enhance the personal interaction of the recipient by creating emotional links between him and the corporate identity. And then the exchange of views and experiences for continuous development and access to distinction and exclusivity.

6- Utilizing the determinants of the knowledge transfer process to develop agreed standards between different cultures to support and circulate them with awareness and harmony; Which contributes to elevating the competitive position of the identity in the local and global markets.

\section{Recommendations:}

1- Paying attention to the application of methods of stimulating the supportive mind through the processes of cultural development and support by encouraging the holding of art exhibitions for different countries, and attending scientific seminars and conferences with the revitalization of translation of international literary works; This is to enrich the conceptual knowledge and circulate it on the largest scale.

2- The designer's investment of all the possibilities available from natural elements, and other values and metaphorical concepts in the design; To enhance reliability and enhance emotional interaction and subjective sensory experience of the recipient.

3- The necessity of developing standards that contribute to the process of knowledge transfer, with the help of specialized experts. To achieve the value of mutual participation between the local and global markets, while adhering to specific controls that ensure the preservation of the national identity of the institution.

\section{Conclusion:}

- Support contributes to the advancement of various cultural, artistic and emotional systems through optimal employment, which is reflected in the enrichment of thought and stimulation of an unconscious response towards sensory, aesthetic and functional values, as well as reviving the cultural heritage with community participation and exchange of experiences between generations and civilizations to achieve hybridization with tight controls based on employing cognitive mechanisms to formulate values And concepts in a modern way in the graphic design elements of the corporate identity. With the aim of presenting a conscious message that affects the consciousness of the recipient and enhances his sensory experience knowledge. 
After presenting the methods of mental support, the researcher concludes how important it is to employ it in the graphic design of the corporate identity for its vital role in reviving the cultural heritage of civilizations and creating links between them by using visual and verbal and other symbols and metaphors in the content of the idea and the advertising message as well as the various types of other arts while applying its dimensions of credibility and enhancing the subjective experiences of the recipient Actively sharing with others; This gives the artistic design a richness and a richness of knowledge that qualifies it to expand its circle from the local to the global in order to remain in the memory of peoples in a vivid and solid way of thinking for long periods, and to invest this in advertising campaigns aimed at promoting tourism and other activities and artistic works.

\section{References:}

1- Batra, R., Seifert, C., \& Brei, D. ( 2016 ). The Psychology of Design:

Creating Consumer Appeal. London \& New York: Routledge.

2- Belussi, F., \& Orsi, L. ( 2016 ). Innovation Alliances, and Networks in High-Tech Environments. London \& New York: Routledge.

3- Caporeal, L. R., Griesmer, J. R., \& Wimsatt W. C. ( 2014 ). Developing Scaffolds in Evolution, Culture, and Cognition. Cambridge, MA: The MIT Press.

4- Colombetti, G., \& Krueger, J. ( 2015 ). Scaffoldings of the affective mind.Psychology Journal, 28 ( 8 ), London \& New York: Routledge, Retrieved from: https://www.tandfonline.com/doi/full/10.1080/09515089.2014.976334

5- Da Rold, F. ( 2018, December ). Defining embodied cognition: The problem situatedness of. New Ideas in Psychology Journal, Vol. ( 51 ). UK: Elsevier. Retrieved April 28, 2018, from: https://www.sciencedirect.com/science/article/abs/pii/S0732118X17301794

6- Dawson, M., Dupuis, B., \& Wilson, M. ( 2010 ). from Bricks to Brains: TheEmbodied Cognitive Science of LEGO Robots. Canada: AU Press, Athabasca University.

7- De Waard, M. ( 2012 ). Imagining Global Amsterdam: History, Culture and Geography in a World City. Amsterdam: Amsterdam University Press.

8- Gracia-Maques, L., Ferreira, M. B. ( 2009 ). The scaffolded mind: The why of emboded cognition ?. European Journal of Social Psychology, Vol. ( 39 ). UK: John Wiley \& Sons. Retrieved from: https://onlinelibrary.wiley.com/doi/pdf/10.1002/ejsp.694

9- Grandori, A. ( 2013 ). Handbook of Economic Organization: Integrating Economic and Organization Theory. Cheltenham, UK: Edward Elgar.

10- Hiniker, A., Hong, R., Kim, Y., Chen, N., West, J. D., \& Aragon, C. ( 2017 ). Toward the Operationalization of Visual Metaphor. Journal of the Association for Information Science 
and Technology. 68 ( 10 ). Hoboken,

11- New Jersey: John Wiley \& Sons. Retrieved October 8, 2016, from: https://onlinelibrary.wiley.com/doi/full/10.1002/asi.23857

12- Kupers, W. M. ( 2013 ). Embodied transformative metaphors and narratives in organisational life-worlds of change. Journal of Organizational Change Management. 26 ( 3 ). UK: Emerald. Retrieved June 26, 2012, from:

https://www.emeraldinsight.com/doi/full/10.1108/09534811311328551

13- Landau, M. J., Meier, B. P., Keefer L. A. ( 2010 ). A Metaphor-Enriched Social Cognition. Psychological Bulletin. 136 ( 6 ). USA: American Psychological Association. Retrieved from: http://psycnet.apa.org/record/2010-18530-001

14- Lee, S. Y., Lim, E. R., \& Drumwright, M. E. ( 2018 ). Hybrid happening: Organizational reputations in corporate crises. Public Relations Review. Vol.(44). UK: Elsevier. Retrieved $\begin{array}{llll}\text { August } 30, & \text { 2017, from: }\end{array}$ https://www.sciencedirect.com/science/article/abs/pii/S0363811117303430

15- Ritzer, G., \& Atalay, Z. ( 2010 ). Readings in Globalization: Key Concepts and Major Debates. UK: John Wiley \& Sons.

16- Suthers, R. A., Fitch, W. T., Fay, R. R., \& Popper, A. N. ( 2016 ). Vertebrate Sound Production and Acoustic Communication. Springer Handbook of Auditory Research. Vol. ( 53 ). Switzerland, Europe: Springer Cham.

17- Vecchi, A. ( 2017 ). Advanced Fashion Technology and Operations Management. Advances in Business Information Systems and Analytics (ABISA) Book Series. USA: IGI Global.

18- Volkenstein, M. V. ( 2011 ). Physical Approaches to Biological Evolution. 2nd Ed. New York: Springer-Verlag Berlin Heidelberg.

19- Yakhlef, A. ( 2007 ). Knowledge transfer as the transformation of context. Journal of High Technology Management Research. 18 ( 1 ). UK: Elsevier.Retrieved from: https://www.sciencedirect.com/science/article/pii/S1047831007000156

20- Youssef, M. ( 2017 ). Culture of Origin as Emotional Catalyst. Master's thesis. Faculty of Applied Arts. Egypt: Helwan university.

21- https://www.behance.net/gallery/72984993/The-package-of-the-cotton-swabs-Art-fict

22- https://blog.beautybridge.com/beauty/7-gift-worthy-holiday-2017-sets-byphilosophy-agiveaway

23- https://brandinginasia.com/starbucks-mooncakes-china/

24- https://creativityawards.com/?submission=omdesign-2017-packaging 
25- https://www.giveacare.ca/

26- http://lalaland-artists.com/artists/norbertschoerner/uniqloheattech/view?id=24037

27- http://www.ms4production.com/projects/uniqlo-heat-tech-lifewear-14/

28- https://nmtesol.files.wordpress.com/2014/05/nmtesol-spring-2014-stone.pdf

29- https://www.packagingoftheworld.com/2018/04/reveur.html

30- https://www.psychologytoday.com/us/blog/conquer-fearflying/201306/emotionalcontrol-top-down-or-bottom

31- https://www.refinery29.com/en-us/how-to-choose-moisturizer?Bucketed=true

32- http://retaildesignblog.net/2018/03/07/i-love-my-mommy-identity-by-yaroslavcherkunov/

Received: February 10, 2018

Accepted: April 28, 2018 